\title{
Two pseudotumor cases mimicking liver malignancy
}

\author{
Mikail Çakır' , Sefa Tüzün'1, Anıl Savaș', Yılmaz Tosyalı²
}

\begin{abstract}
Inflammatory pseudotumor of the liver is a rare benign tumor that can be confused with malignant tumors of the liver. It is usually diagnosed after pathologic evaluation of the resected lesion. If a mass lesion in the liver is suspicious for malignancy on radiologic evaluation then surgical resection is planned for suitable patients rather than a biopsy. Inflammatory pseudotumors are similar to malignant tumors on macroscopic assessment, but microscopically they are characterized by the presence of inflammatory cells. In case of clinical or radiologic suspicion, the lesion is biopsied and once the diagnosis of inflammatory pseudotumor is made the lesion is managed by conservative-medical treatment. It must be kept in mind as part of differential diagnosis to avoid unnecessary surgery.
\end{abstract}

Keywords: Liver tumor, inflammatory pseudotumor, liver resection

\section{INTRODUCTION}

Inflammatory pseudotumor of the liver (IPT) is a rare benign tumor that can be confused with malignant liver tumors. The most frequent occurrence sites except the liver include the central nervous system, intestine, omentum, orbit, urinary bladder, breast, pancreas, lymph nodes, skin and soft tissue (1). Macroscopically, the lesions may mimic malignancy, the nodules can be solitary or multiple. Their size may reach up to $25 \mathrm{~cm}$. Microscopically, they are characterized by fibrovascular tissue proliferation, spindleshaped cells, myofibroblasts, and mixed inflammatory cells (plasma cells, lymphocytes, histiocytes, eosinophils) (2). A retrospective analysis of 403 patients operated on for focal liver lesions revealed an IPT incidence of $0.7 \%$ (3). There are fewer than 300 cases reported in the literature (4). In this article, we present two patients who have undergone right and left hepatectomy, respectively, with a pre-operative diagnosis of malignancy who were diagnosed with inflammatory pseudotumor of the liver on pathologic examination.

\section{CASE PRESENTATIONS}

\section{Case 1}

Fibronecrotic granulomatous inflammation mimicking hepatocellular carcinoma- underwent right hepatectomy.

Cite this paper as:

Çakır M, Tüzün S, Savaş A, Tosyalı Y. Two pseudotumor cases mimicking liver malignancy. Turk J Surg 2017; 33: 212-216.

'Clinic of General Surgery, Haseki Training and Research Hospital, İstanbul, Turkey

${ }^{2}$ Clinic of Pathology, Haseki Training and Research Hospital, İstanbul, Turkey

Address for Correspondence Mikail Çakır

e-mail:

drmikailcakir@yahoo.com

Received: 17.09.2014

Accepted: 12.02.2015

Available Online Date: 02.07.2015

cCopyright 2017

by Turkish Surgical Association www.turkjsurg.com

A 58-year-old man who has been followed-up for type 2 diabetes mellitus for eight years underwent abdominal ultrasound for evaluation of a $5 \mathrm{~kg}$ weight loss within the past 6 months that revealed a $5 \mathrm{~cm}$ mass in the $5^{\text {th }}$ segment of the liver. His abdominal computed tomography (CT) and magnetic resonance imaging (MRI) revealed radiological findings suggesting hepatocellular carcinoma (HCC) with a hypervascular $5 \mathrm{~cm}$ mass in the $5^{\text {th }}$ segment extending to the $6^{\text {th }}$ segment with early washout (Figure 1,2$)$. The patient was planned for right hepatectomy.

Laboratory findings: Aspartate aminotransferase (AST): $12 \mathrm{U} / \mathrm{L}$, alanine aminotransferase (ALT): $8 \mathrm{U} / \mathrm{L}$, gamaglutamyl transferase (GGT): $19 \mathrm{U} / \mathrm{L}$, alkaline phosphatase (ALP): $85 \mathrm{U} / \mathrm{L}$, Total bilirubin (T.Bil): $0.6 \mathrm{~g} /$ $\mathrm{dL}$, direct bilirubin (D.Bil): $0.1 \mathrm{mg} / \mathrm{dL}$, INR: 0.9, tumor markers: CA 19-9: $2.7 \mathrm{U} / \mathrm{mL}$, AFP: 2,6 ng/mL, Hepatitis markers: HBsAg: (-), Anti Hbs: (+), Anti HCV: (-).

His positron emission tomography (PET-CT) showed heterogeneous moderately increased pathological fluorodeoxyglucose (FDG) uptake in the $5^{\text {th }}-6^{\text {th }}$ segments of the liver (early SUVmax: 4.6, late SUVmax: 5.8). The partial increase in FDG uptake in late images was interpreted in favor of HCC (Figure 3).

The patient underwent right hepatectomy after obtaining informed consent. He was followed-up in the intensive care unit for 12 days due to lung infection and atelectasis in the postoperative (PO) period. Oral intake was started on the $3^{\text {rd }}$ PO day. He was admitted to the clinic on the 12th PO day with 
approximately $1000 \mathrm{cc}$ of ascites drainage per day. The ascites was kept under control with albumin, furosemide, spironolactone and enteral hyperalimentation. His bilirubin levels increased starting from the $2^{\text {nd }} \mathrm{PO}$ day. His laboratory results on PO day 12 were as follows: AST: 113 U/L, ALT: 82 U/L, ALP: 375

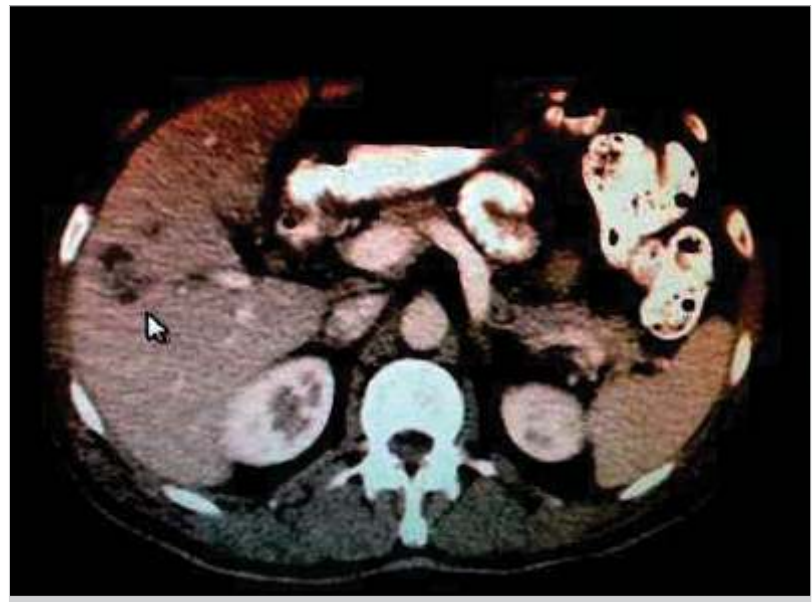

Figure 1. First patient, HCC appearance in CT $\mathrm{CT}$ : computed tomography; HCC: hepatocellular carcinoma

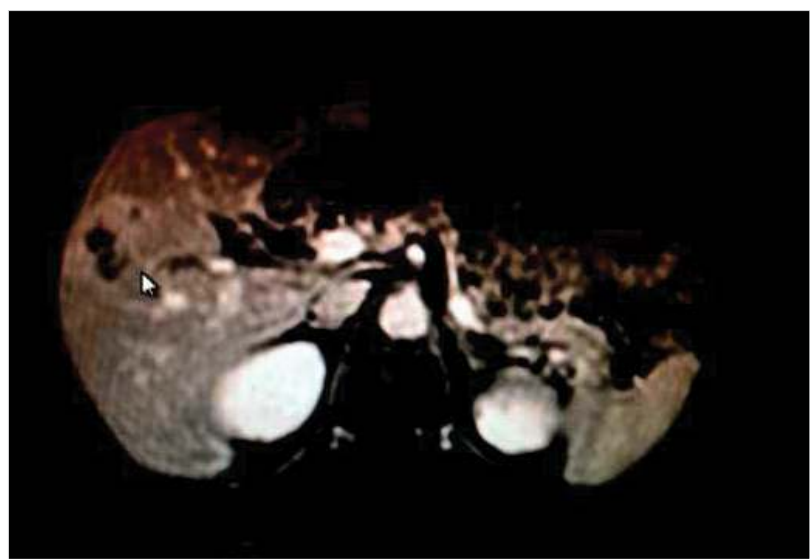

Figure 2. First patient, HCC appearance in MRI MRI: magnetic resonance imaging; HCC: hepatocellular carcinoma

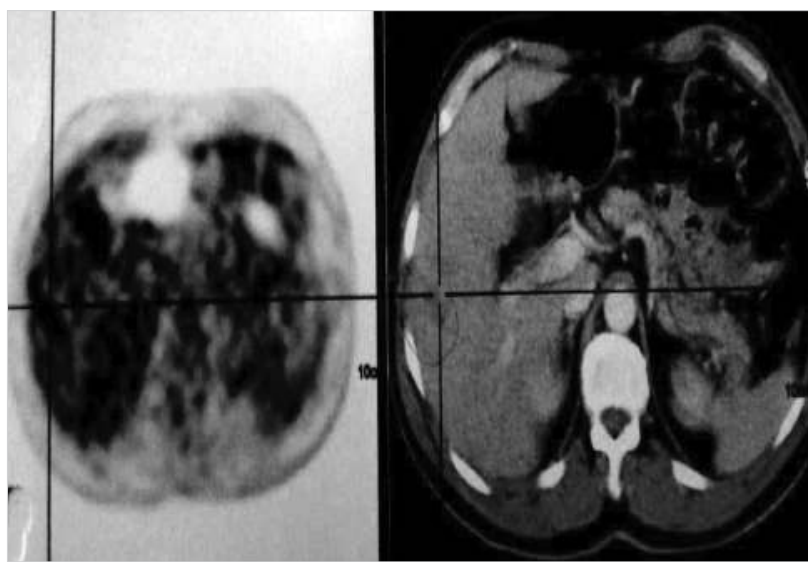

Figure 3. PET CT scan of the first patient: Heterogeneous moderate increased pathologic FDG uptake in the 5th-6th segments of the liver (Early SUVmax: 4.6, Late SUVmaks: 5.8). Interpreted as HCC

PET CT: positron emission tomography computed tomography; FDG: fluorodeoxyglucose; HCC: hepatocellular carcinoma
U/L, GGT: 567 U/L, T. Bil:11 mg/dL, D. Bil: 6 mg/dL, INR: 1.3 Total Protein (T.Pro): $4.4 \mathrm{~g} / \mathrm{dL}$, Albumin (Alb): $2.9 \mathrm{~g} / \mathrm{dL}$. The bilirubin level peaked at $20 \mathrm{mg} / \mathrm{dL}$ (AST: $212 \mathrm{U} / \mathrm{L}$, ALT: $172 \mathrm{U} / \mathrm{L}$, ALP: 261 U/L, GGT: $295 \mathrm{U} / \mathrm{L}$ ) on the $30^{\text {th }} \mathrm{PO}$ day. His MR and MR cholangiography (MRCP) did not reveal intrahepatic or extrahepatic biliary dilation. There were ischemic changes in the 4th segment and the caudate lobe. The patient was considered as hepatic insufficiency at regeneration stage. The bilirubin levels began decreasing after reaching $20 \mathrm{mg} / \mathrm{dL}$.

The patient was discharged on the $36^{\text {th }}$ postoperative day. His biochemical parameters were completely normal in the second month follow-up. The patient is being followed-up for one year with no additional problems.

Pathology: The patient's pathology evaluation showed fibronecrotic granulomatous inflammatory reaction (Figure 4). The further evaluation for infectious and granulomatous diseases did not reveal any specific etiology.

\section{Case 2}

Xanthogranulomatous inflammation with cholangitis mimicking cholangiocellular carcinoma- underwent left hepatectomy.

A 53-year-old male patient was admitted to the emergency department with complaints of jaundice, abdominal pain and fever, and was diagnosed with cholangitis. His past medical history included coronary bypass operation 7 years ago and insulin-dependent diabetes mellitus for 10 years.

Laboratory findings: White blood cell (WBC): 4600, Hematocrit (Hct): 42\%, T. Bil: 10.4 mg/dL, D. Bil: 5.9 mg/dL, AST: 143 U/L, ALT. 228 U/L, ALP 257 U/L, GGT: 959 U/L, INR: 0.8, CA19-9: $1254 \mathrm{U} / \mathrm{mL}$.

Our case presented with cholangitis and was initially evaluated as choledocholithiasis-associated jaundice. The emergency US revealed intrahepatic biliary dilation mainly in the left lobe, the common bile duct was $6.3 \mathrm{~mm}$ in diameter, the gallbladder was contracted. His MR-MRCP showed distal common bile duct benign stricture, impacted stone, intrahepatic

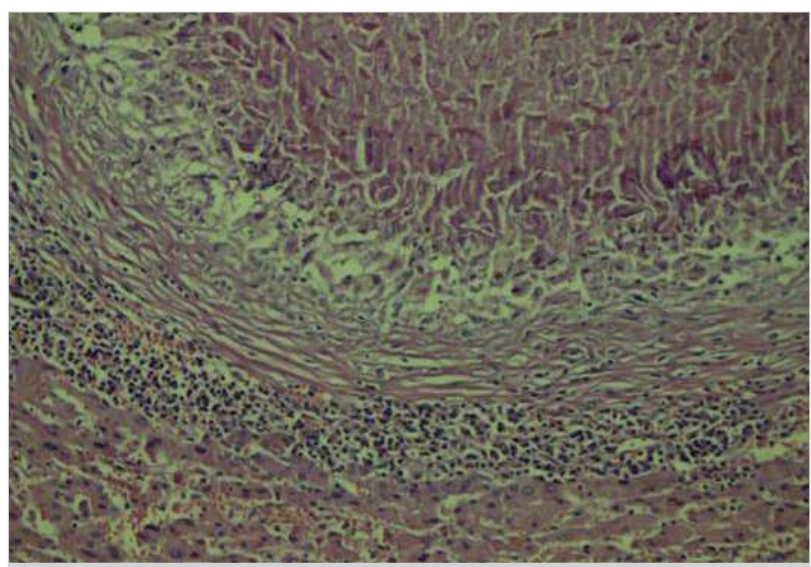

Figure 4. Pathology image of the first case: Periportal areas enlarged due to inflammation. Fibronecrotic granulomatous foci associated with bile ducts and progressing into the parenchyma are observed. Different sized foci form aggregates (HE x100). 
biliary dilation, and abscess mass in the left lobe (thought to be secondary to cholangitis). An endoscopic retrograde cholangiopancreatography (ERCP) was performed, with abundant sludge and microcalculi drainage following endoscopic sphincterotomy. There was no stricture that obstructed contrast passage (contradictory to MRCP findings). Intrahepatic bile ducts could not be evaluated in detail. Bilirubin values and cholestasis enzymes were slightly decreased, but there was no significant improvement. When the patient was assessed further by CT scan, the contrast enhancement pattern of the mass was found to be consistent with cholangiocellular carcinoma (Figure 5, 6). The PET-CT showed heterogeneous, intense pathologic FDG uptake (SUVmax: 17.4) with biliary tract dilation in nearly the entire left hepatic lobe. The right lobe was preserved and the findings were interpreted as cholangiocellular carcinoma (Figure 7).

He was scheduled for left hepatectomy after obtaining informed consent. In the operation, there was extensive inflam-

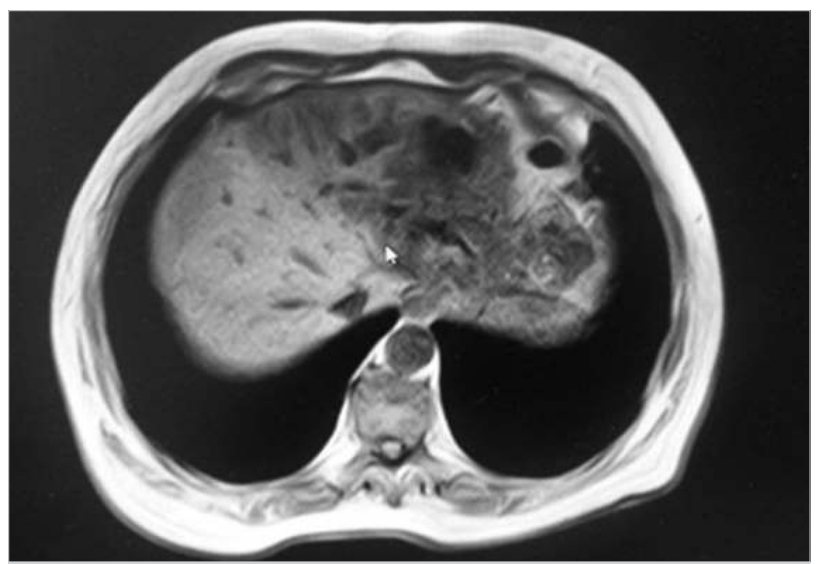

Figure 5. MR-MRCP in the second patient. A mass accompanied by $3 \times 2 \mathrm{~cm}$ perfusion defect in the 4 a segment, with a significant pre-mass IHBD dilation. Findings consistent with central dilated cholangiocellular carcinoma or focal bile duct dilation due to Caroli disease. Common hepatic and common bile duct are normal. Right IHBD are normal.

MR-MRCP: magnetic resonance-magnetic resonance cholangiopancreatography; IHBD: intrahepatic bile ducts

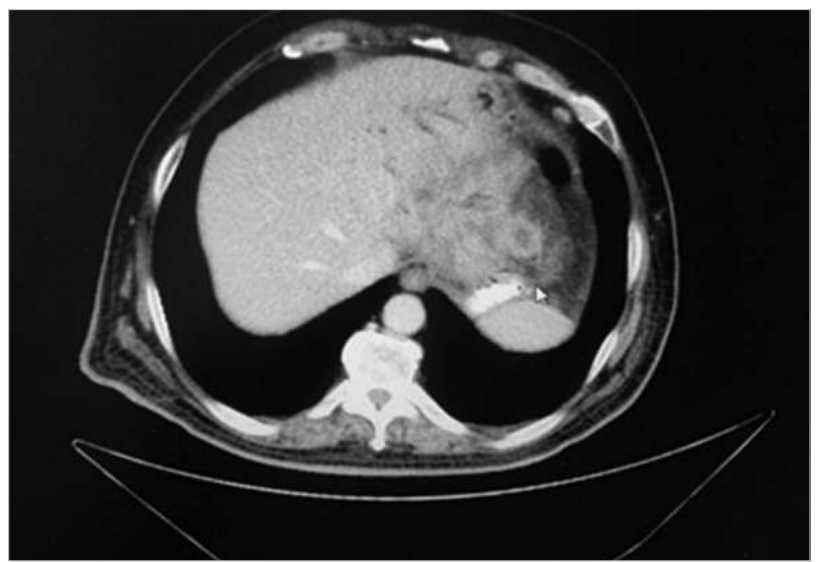

Figure 6. Abdominal CT-CT angiography of the second patient: $3 \mathrm{~cm}$ hypodense area in segment $4 \mathrm{a}$ accompanied by marked IHBD dilation in the left lobe mation in the liver as well as lesions suggesting metastatic nodules in both the left and right hepatic lobes. The frozensection evaluation of these nodules did not show apparent malignancy, nevertheless, since the suspicion of malignancy persisted, numerous biopsies were obtained and the operation was terminated. Since malignancy could not be ruled out even on biopsy materials, left hepatectomy was performed one week later.

Pathology: Pathologic evaluation was concluded as inflammatory pseudotumor secondary to cholangitis.

\section{General surgery follow-up}

Preoperative final laboratory values: AST: $106 \mathrm{U} / \mathrm{L}, \mathrm{ALT}: 66 \mathrm{U} / \mathrm{L}$, GGT: 184 U/L, ALP: 362 U/L, T. Bil: 14 mg/dL, D.Bil: 9 mg/dL, T.Pro: 6.5 g/dL, Alb: 2.2 g/dL, INR: 1.1, Ca 19-9: 362 U/mL, Hct: 27\%, WBC: 9000.

The patient was planned for left hepatectomy with a presumptive diagnosis of cholangiocellular carcinoma.

First surgery: The patient was taken to surgery after obtaining informed consent. A small amount of ascites was detected on exploration and samples were taken for cytopathological and biochemical analyzes. The liver was completely inflamed with tumor nodules in the left lobe $2^{\text {nd }}$ and $4^{\text {th }}$ segments, and there were a few $1-\mathrm{cm}$ nodular lesions suggesting metastasis in the right lobe $5^{\text {th }}$ and $7^{\text {th }}$ segments. Biopsies were obtained from segments 2, 3, 5 and 7. On frozen-section examination, the lesions were not evaluated as malignancy but as fibrosis. Since intrahepatic metastases could not be ruled out, the operation was terminated to await paraffin section examination results, which revealed active chronic inflammation, fibrosis, biliary duct proliferation, cholangitis, lobular distortion, presence of bile pigment in hepatocytes, and balloon degeneration. Ascites evaluation did not reveal any specific feature.

Conclusion: The findings were interpreted as changes secondary to bile duct obstructive neoplasia. Malignancy could not be ruled out.

As the samples obtained from the right liver did not show signs of metastasis, and the possibility of malignancy of the left lobe mass continued, the patient was scheduled for left hepatectomy one week later. Left hepatectomy was performed in the inflamed and edematous liver.

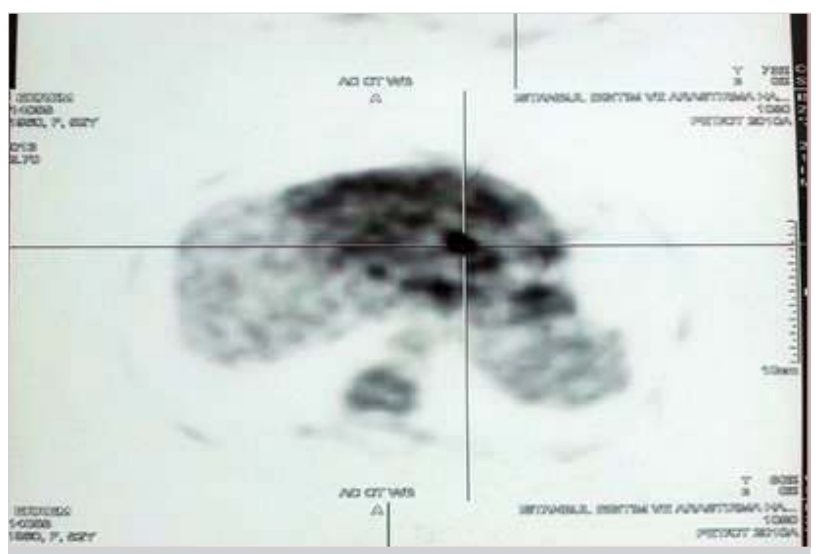

Figure 7. PET CT findings compatible with cholangiocellular carcinoma of the second patient.

PET CT: positron emission tomography computed tomography 
Second pathology: On gross examination, there were extensive nodular infiltration areas of $0.5-1 \mathrm{~cm}$ in size with nodules up to $6 \times 3 \mathrm{~cm}$ in segment 3 , and of $5 \times 3 \mathrm{~cm}$ in segment 5 . The surgical margin was negative by $1.5 \mathrm{~cm}$.

Microscopic findings: Active chronic cholangitis with extensive bile duct destruction, xanthogranulomatous inflammation-inflammatory pseudotumor findings replacing the parenchyma (Figure 8), chronic pericholecystitis forming lymphoid aggregates in the cholecystectomy material, reactive hyperplasia in dissected lymph nodes.

Clinical course: The patient was followed-up in the intensive care after both operations, and remained there for 2 days after the first surgery and 3 days after the second. He developed wound infection and ascites drainage in the postoperative period. Wound infection was controlled with antibiotics, ascites drainage was treated with albumin, furosemide, and spironolactone. There was no specific finding in his outpatient work-up.

\section{DISCUSSION}

The etiology of inflammatory pseudotumor of the liver include infectious agents (Bacteriodes caccae, Actinomyces, Klebsiella, E. coli), autoimmune hepatopancreatobiliary diseases (IgG4 sclerosing cholangitis), and systemic granulomatous diseases (tuberculosis, sarcoidosis) (5).

Patients present with complaints of fever, abdominal pain, weight loss, jaundice (secondary to obstruction of intra- or extra-hepatic bile ducts by the inflammatory mass). Laboratory and radiological findings are not very specific for differential diagnosis. Biopsies may be performed to avoid unnecessary surgical intervention in suspicious contrast enhancement of the liver lesion, and in positive laboratory findings of infection, inflammation, or autoimmune diseases (6). Biopsy has complications such as bleeding, tumor seeding, and infection. Therefore, masses suggesting liver malignant tumors with typical

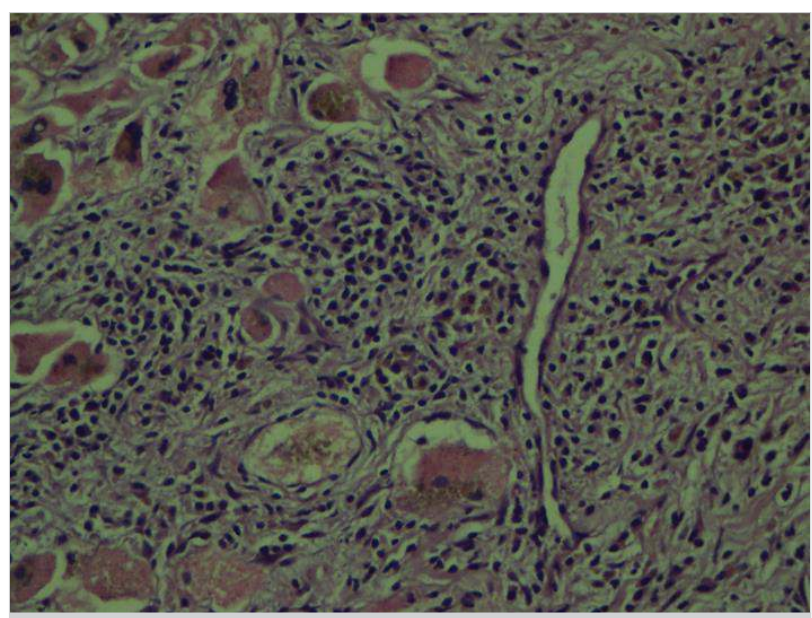

Figure 8. Pathologic view of the second patient. Diffuse cell infiltration consists of lymphocytes, plasmocytes, eosinophils and neutrophil polymorphs in each area. Cell infiltration is concentrated around periportal areas and ducts, and extends into the parenchyma. Bile ducts are proliferated. Bile pigment accumulation and balloon degeneration are evident in hepatocytes. Polymorphs form aggregates in several areas (HE x400). contrast-enhancement pattern are treated by resection without biopsy. Biopsies are performed due to suspicion in masses that do not suggest malignancy by contrast-enhancement pattern (4).

Patients diagnosed with pseudotumor on biopsy are treated with antibiotics, non-steroidal anti-inflammatory drugs, and corticosteroids. Constitutional symptoms and lesion regression are closely monitored $(4,7)$. Surgery is considered if conservative treatment fails (persistent fever, tumor enlargement) or in case of hepatic hilum involvement, jaundice or portal hypertension (8).

In most cases, IPT is confused with other malignant tumors and is diagnosed pathologically after surgery. Surgery should be considered in case of diagnostic uncertainty and ongoing tumor suspicion (7).

Although several reports indicate no recurrence after surgical resection, there are reports on malignant transformation and recurrences even 4-7 years later $(7,10)$.

\section{CONCLUSION}

Inflammatory pseudotumor of the liver is a very rare condition that can be confused with malignant tumors. It is associated with infectious, inflammatory and autoimmune diseases. The tumor appearance in the liver shows contrast-enhancement pattern similar to other malignancies. IPT should be kept in mind as part of differential diagnosis in patients with systemic symptoms, laboratory or imaging findings, and unnecessary surgery should be avoided by biopsy in case of suspicion. They should be treated with conservative medical treatment (antibiotics, anti-inflammatory drugs, corticosteroids) and surgery should be applied in unresponsiveness to treatment and suspicion for malignancy. Most cases are diagnosed with pathologic evaluation after resection of a mass due to suspicion of malignancy. There are reports of recurrence and longterm malignant transformation (10), thus patients should be followed-up closely.

Informed Consent: Written informed consent was obtained from patients who participated in this case.

Peer-review: Externally peer-reviewed.

Author Contributions: Concept - M.Ç.; Design - A.S.; Supervision M.Ç.; Funding - M.Ç.; Materials - M.Ç., S.T.; Data Collection and/or Processing - A.S., M.Ç.; Analysis and/or Interpretation - M.Ç., A.S.; Literature Review - A.S.; Writer - M.Ç.; Critical Review - S.T.

Conflict of Interest: No conflict of interest was declared by the authors.

Financial Disclosure: The authors declared that this study has received no financial support.

\section{REFERENCES}

1. Ntinas A, Kardassis D, Miliaras D, Tsinoglou K, Dimitriades A, Vrochides $D$. Inflammatory pseudotumor of the liver: a case report and review of the literature. J Med Case Rep 2011; 5: 196. [CrossRef]

2. Someren A. Inflammatory pseudotumor of liver with occlusive phlebitis: report of a case in child and review of the literature. Am J Clin Pathol 1978; 69: 176-181. [CrossRef] 
3. Tang L, Lai EC, Cong WM, Li AJ, Fu SY, Pan ZY, et al. Inflammatory myofibroblastic tumor of the liver: a cohort study. World J Surg 2010; 34: 309-313. [CrossRef]

4. Calomeni GD, Ataíde EB, Machado RR, Escanhoela CA, Costa LB, Boin IF. Hepatic inflammatory pseudotumor: A case series. Int J Surg Case Rep 2013; 4: 308-311. [CrossRef]

5. Goldsmith PJ, Loganathan A, Jacob M, Ahmad N, Toogood GJ, Lodge JP, et al. Inflammatory pseudotumours of the liver: a spectrum of presentation and management options. Eur J Surg Oncol 2009; 35: 1295-1298. [CrossRef]

6. Kato H, Kanematsu M, Kondo H, Osada S, Goshima S, Yamada T, et al. Inflammatory pseudotumor of the liver: ferumoxide-enhanced MR imaging as a tiebreaker. J Magn Reson Imaging 2004; 20: 501-505. [CrossRef]
7. Kim YW, Lee JG, Kim KS, Yoon DS, Lee WJ, Kim BR, et al. Inflammatory pseudotumor of the liver treated by hepatic resection: a case report. Yonsei Med J 2006; 47: 140-143. [CrossRef]

8. Kaneko K, Ando H, Watanabe $\mathrm{Y}$, Seo T, Nagino M, Kamiya J, et al. Aggressive preoperative management and extended surgery for inflammatory pseudotumor involving the hepatic hilum in a child. Surgery 2001; 129: 757-760. [CrossRef]

9. Ke Q, Fan L, Duan X, He Z, Zheng S. Inflammatory pseudotumor mimicking primary hepatic malignant tumor with hepatitis $B$ virus-related cirrhosis: A case report. Oncol Lett 2013; 6: 550-552. [CrossRef]

10. Pecorella I, Ciardi A, Memeo L, Trombetta G, de Quarto A, de Simone $\mathrm{P}$, et al. Inflammatory pseudotumour of the liver--evidence for malignant transformation. Pathol Res Pract 1999; 195: 115-120.[CrossRef] 